\title{
Narrative review: update on immunotherapy and pathological features in patients with bladder cancer
}

\author{
Gaetano Aurilio $^{1 \#}$, Alessia Cimadamore ${ }^{2 \#}$, Antonio Lopez-Beltran ${ }^{3}$, Marina Scarpelli ${ }^{2}$, Francesco Massari ${ }^{4}$, \\ Elena Verri ${ }^{1}$, Liang Cheng ${ }^{5}$, Matteo Santoni ${ }^{6}$, Rodolfo Montironi ${ }^{2}$ \\ ${ }^{1}$ Medical Oncology Division of Urogenital and Head and Neck Tumours, IEO, European Institute of Oncology IRCCS, Milan, Italy; ${ }^{2}$ Section of \\ Pathological Anatomy, United Hospitals, School of Medicine, Polytechnic University of the Marche Region, Ancona, Italy; ${ }^{3}$ Department of Surgery, \\ Cordoba University Medical School, Cordoba, Spain; ${ }^{4}$ Medical Oncology, IRCCS Azienda Ospedaliero-Universitaria di Bologna, Bologna, Italy; \\ ${ }^{5}$ Department of Pathology and Laboratory Medicine, Indiana University School of Medicine, Indianapolis, IN, USA; ${ }^{6}$ Oncology Unit, Macerata \\ Hospital, Macerata, Italy \\ Contributions: (I) Conception and design: Gaetano Aurilio, Alessia Cimadamore, Rodolfo Montironi; (II) Administrative support: Rodolfo Montironi; \\ (III) Provision of study materials or patients: None; (IV) Collection and assembly of data: G Aurilio, A Cimadamore, E Verri; (V) Data analysis and \\ interpretation: Antonio Lopez-Beltran, Liang Cheng, Francesco Massari, M Santoni, M Scarpelli; (VI) Manuscript writing: All authors; (VII) Final \\ approval of manuscript: All authors. \\ \#These authors contributed equally to this work. \\ Correspondence to: Alessia Cimadamore. Pathological Anatomy, United Hospitals, School of Medicine, Polytechnic University of the Marche Region, \\ Via Conca 71, I-60126 Ancona, Italy. Email: a.cimadamore@staff.univpm.it.
}

\begin{abstract}
Over the last few years efficacy of immunotherapy using immune checkpoint inhibitors (ICI) has been investigated in patients with bladder cancer (BC) at all stages. The present article aims to assess new therapeutic options with emerging agents in BC patients, shedding light on ICI-based treatments encompassing all disease stages, from non-muscle invasive (NMIBC) to muscle-invasive (MIBC) BC, concluding with metastatic MIBC. In bacillus Calmette-Guerin (BCG) unresponsive patients with carcinoma in situ, pembrolizumab has been recently approved. In the neoadjuvant setting, results from two clinical trials seem to identify pathological and genomic features of highly responsive tumors. Squamous cells and lymphoepithelioma/like histotypes, programmed cell-death ligand 1 (PD-L1) expression and high levels of activate $T$ cells have been associated with higher response rate. In the metastatic setting, only $30 \%$ of patient may respond to ICI. A panel of biomarkers for patient selection is an actual need since the correlation between response and PD-L1 expression seem inconsistent across clinical trials, with some exceptions. Molecular characterization of BC, tumor mutation burden and immune-gene expression profiling might introduce new molecular biomarkers, hopefully transferable into the clinical-pathological practice.
\end{abstract}

Keywords: Urothelial carcinoma; bladder cancer (BC); immune checkpoint inhibitors (ICIs); immunotherapy; predictive biomarkers; programmed cell-death ligand 1 (PD-L1); clinical trials

Submitted Nov 18, 2020. Accepted for publication Jan 27, 2021.

doi: $10.21037 /$ tau-20-1436

View this article at: http://dx.doi.org/10.21037/tau-20-1436

\section{Introduction}

Bladder cancer (BC) is the $11^{\text {th }}$ most commonly diagnosed cancer worldwide $(1,2)$. The vast majority of patients with $\mathrm{BC}$ presents with early stage [stage Ta/carcinoma in situ (CIS) or T1]. The 5 -year survival rates vary from $98 \%$ in stage Ta, to $20.9 \%$ in case of lymph node spread to $6.8 \%$ in patients with visceral metastases (3).

Chemotherapy with platinum-based regimens has always been the cornerstone of medical treatment of BC patients, either as perioperative strategy and/or as front-line therapy for locally advanced or stage IV metastatic disease. 
However, after failure of platinum-based chemotherapy, effective therapeutic options are limited and prognosis is very poor for these patients. Over the last few years efficacy of immunotherapy using immune checkpoint inhibitors (ICIs) has been investigated in patients with BC at all stages. Indeed, anti-programmed cell-death protein 1 (PD-1) pembrolizumab and nivolumab, as well as antiprogrammed cell-death ligand 1 (PD-L1) durvalumab, avelumab, and atezolizumab, have been labeled by the Food and Drug Administration (FDA) for metastatic urothelial BC patients who had disease progression after platinum-based chemotherapy. The present article aims to assess new therapeutic options with emerging agents in BC patients, shedding light on ICI-based treatments encompassing all disease stages, from non-muscle invasive (NMIBC) to muscle-invasive (MIBC) BC, concluding with metastatic MIBC.

\section{Article selection}

We performed a comprehensive literature search of the PubMed/Medline database focusing on the keywords "bladder cancer", "urothelial carcinoma", "immune checkpoint inhibitors", "immunotherapy", "predictive biomarkers", "outcome", and "clinical trials". Most relevant studies in terms of number of patients and clinical impact were selected. The manuscript is presented in accordance with Narrative Review reporting checklist (available at http://dx.doi.org/10.21037/tau-20-1436).

\section{Narrative review}

\section{ICI clinical trials from NMIBC to non-metastatic MIBC}

Intravesical immunotherapy with bacillus Calmette-Guerin (BCG) is the standard treatment to prevent the recurrence in intermediate/high-risk NMIBC (4). However, $40 \%$ of patients treated with BCG will develop disease recurrence. Early radical cystectomy (RC) is strongly recommended in patients with BCG unresponsive tumors (5). Bladdersparing treatments are currently under investigation. In this setting, PD-1/PD-L1 inhibitors combined with or without BCG are being tested in NMIBC patients, with encouraging results. The rationale behind this is that PD-L1 expression has been associated with increased resistance to BCG immunotherapy. Moreover, granulomas induced by BCG in BCG-unresponsive patients show high levels of PD-L1 expression. The high expression of PD-L1 may suppress the T-cell response induced by BCG and be the cause of the BCG failure (6). A recent publication by Pierconti et al. examined the expression of PD-L1 both in tumor cells and in immune cells in patients with CIS primary responders and not responders to BCG therapy. According to their results, $\mathrm{PD}-\mathrm{L} 1$ positive expression performed using the $22 \mathrm{C} 3$ clone identify patients with BCG-unresponsive CIS (7).

Pembrolizumab in BCG-unresponsive NMIBC patients The KEYNOTE-057, phase II, is the first single-arm study enrolling patients with BCG unresponsive disease to receive pembrolizumab administered intravenously (IV) at a dose of $200 \mathrm{mg}$ every 3 weeks for 24 months or until disease progression or discontinuation due to toxicity. Preliminary data on 103 NMIBC patients with high-risk BCGunresponsive CIS with/without papillary disease (cohort A) not suitable for RC have been reported in abstract form. The findings showed a 3 -month complete response rate of $38 \%$, with a lasting response in $72 \%$ of the patients. Nineteen patients (18\%) experienced immune-mediated adverse events, one of these (colitis) led to a toxic death (8).

Based on these preliminary data, the FDA granted the approval of Pembrolizumab for the treatment of BCG unresponsive patients with CIS with/without papillary tumors who are ineligible for or elected not to undergo RC.

Currently ongoing clinical trials are testing durvalumab (NCT02901548) in patients with BCG refractory CIS, atezolizumab for BCG-unresponsive high-risk NMIBC (Ta/T1/CIS) (NCT02844816) and as neoadjuvant therapy to patients with either BCG-refractory NMIBC, or MIBC appropriate for RC and refusing or ineligible for neoadjuvant chemotherapy (NCT02451423). Phase I studies are now investigating the safety and preliminary antitumor activity of atezolizumab and pembrolizumab in combination with intravesical BCG (NCT02792192, NCT02324582). Five trials are currently investigating the use of ICI in BCG untreated patients but no results available yet.

Predictive biomarkers of response are needed in this setting. Molecular analysis comparing genomic profiles of BCG responders and BCG treated patients who progressed to MIBC evidenced a significant reduction of tumor mutational burden (TMB) in responders compared to "progressors" and metastatic disease. More advanced tumors express a lower burden of neoantigens that may explain the decreased response to BCG immunotherapy (9).

Since the first molecular taxonomy published by Sjödahl et al. in 2012 (10), several attempts have been made trying 
to classify the NMIBC in different genomic-based groups $(11,12)$. The latest one, recently published by Robertson et al. (13), aimed to investigate the molecular heterogeneity of T1 urothelial cancer with a primary endpoint of recurrence after BCG. Five consensus subtypes of T1 tumors treated with BCG were identified. The so-called T1-Myc and T1-early subtypes had the significantly worse recurrence-free survival than the other three subtypes grouped. Interestingly, both subtypes had elevated MYC expression and T1-early had a repressed immune response hallmarks for IFN- $\alpha$ and IFN- $\gamma$ suggesting its tumor microenvironment may represent an immune desert.

\section{Perioperative immune trials in MIBC patients}

Several immunotherapy-based trials are in clinical development in patients with localized MIBC, either as immuno-based combination strategies or as ICI single-agent, though data are available just for two neoadjuvant phase II trials testing atezolizumab (14) and pembrolizumab (15), respectively.

The ABACUS is a phase II study in MIBC cisplatinunfit patients with clinical T2-T4aN0 stage tumor, planned to assess the efficacy of atezolizumab as neoadjuvant treatment. A total of 95 participants have received threeweekly atezolizumab IV at a dose of $1,200 \mathrm{mg}$ for 2 cycles, prior to RC. Pathologic complete remission (pT0) was observed in $31 \%$ of the patients, rate that increased to $37 \%$ in $\mathrm{PD}-\mathrm{L} 1$ positive patients. The most common adverse event was fatigue in $21 \%$ of the patients (14). The PURE01 is a phase II, single-arm study, that has investigated the activity of pembrolizumab before $\mathrm{RC}$ in predominant urothelial cisplatin-fit MIBC patients with clinical $\mathrm{T} \leq$ $3 \mathrm{bN0}$ stage tumor. Fifty patients were planned to receive three-weekly pembrolizumab IV at a dose of $200 \mathrm{mg}$ for 3 cycles. The results demonstrated overall that 21 out of $50(42 \%)$ patients got a pT0 stage; in 35 patients with PDL1 combined positive score (CPS) $\geq 10 \%$ a total of 19 (54\%) experienced a pT0 response. Thyroid dysfunction was the most frequent all-grade toxicity and occurred in $18 \%$ of the patients. Only one patient interrupted the study drug for grade 3 transaminitis (15).

In the ABACUS trial, $\mathrm{PD}-\mathrm{L} 1$ expression was considered positive if present in $\geq 5 \%$ of immune cells using the SP142 antibody. No significant correlation between PDL1 expression and outcome, on either immune cells or tumor cells was found $(14,16)$. Notably, preexisting $T$ cell activation correlated with response. A higher percentage of inflamed tumors compared to metastatic setting was reported; among inflamed tumors, responding tumors had an inflammatory infiltrate composed by $\mathrm{T}$ cells double positive for CD8 and granzyme B (a surrogate marker for CD8 activated cells) unlike relapsing inflamed tumors, which showed low levels of activated T cells. TMB, on the contrary, did not correlate with response.

Of interest, in the PURE-01 trial, patients with squamouscell carcinoma or a lymphoepithelioma-like variant of $\mathrm{BC}$ had major, although preliminary, pathological responses compared with those with other predominant variant histologies. PDL1 expression was evaluated with 22C3 antibody using CPS. $\mathrm{TMB}$ and CPS were associated with both $\mathrm{pT} 0$ and $\mathrm{pT} \leq 1$ responses, irrespective of tumor histology (17).

\section{ICI clinical trials in metastatic MIBC}

\section{Atezolizumab as first-line treatment}

IMvigor210 is a single-arm, multicentre, phase 2 trial of atezolizumab as front-line therapy in untreated cisplatinineligible MIBC patients. A total of 123 patients were enrolled and 119 of whom received atezolizumab IV at a dose of 1,200 mg every 3 weeks until disease progression occurred. The main reason for cisplatin-unfit condition was renal impairment in $70 \%$ of the treated patients. At a median follow-up of 17 months, 27 out of 119 (23\%) patients experienced an objective response rate (ORR), $11(9 \%)$ of whom as complete remission, for an overall clinical benefit rate of $30 \%$. Clinical responses there were both early ( 2 months) than late (after 6 months) during atezolizumab treatment, and were observed across all PDL1 subgroups according to tumour-infiltrating immune cells status and by baseline prognostic subgroups. In all patient population, median progression-free survival (PFS) was 2.7 months that rose to 4.1 months in patients with immune cells score of $2 / 3$, while median overall survival (OS) was equal to 15.9 months and increased to 19.1 months in patients with immune cells score of $0 / 1$. Atezolizumab toxicity was well-tolerated with manageable immune-mediated events that required steroid therapy alone, only one toxic death for sepsis occurred (18). Of notice, the luminal I subtype had lower PD-L1 immune cell expression and $\mathrm{CD} 8^{+}$genes expression, thus achieving lower response rates (19).

IMvigor130 is a multicentre, placebo-controlled, phase 3 trial of atezolizumab with or without chemotherapy as first-line treatment in patients with locally advanced or metastatic urothelial MIBC. The trial allowed the inclusion of patients with or without a cisplatin-eligibility condition. 
A total of 1,213 patients were randomized (1:1:1) to receive atezolizumab 1,200 mg IV every 3 weeks with platinumbased chemotherapy (cohort $\mathrm{A}, \mathrm{n}=453$ ), or atezolizumab $1,200 \mathrm{mg}$ IV as single-agent therapy (cohort $\mathrm{B}, \mathrm{n}=354$ ), or placebo with platinum-based chemotherapy (cohort C, $\mathrm{n}=390$ ). At a median follow-up of 11.8 months, median primary PFS resulted equal to 8.2 months in cohort A versus 6.3 months in cohort $\mathrm{C}(\mathrm{P}=0.007)$. Interim median OS findings showed an improvement of 16 months for cohort A versus 13.4 months for cohort $\mathrm{C}(\mathrm{P}=0.027$, value that did not meet the preplanned threshold of statistical significance), and 15.7 months for cohort B versus 13.1 months for cohort C. Treatment-related serious adverse events were reported in $32 \%$ of patients in cohort A, $12 \%$ in cohort B, and $26 \%$ in cohort C. Atezolizumabrelated grade 3 or 4 adverse events of special interest occurred in $8 \%$ of patients both in cohort A than in cohort B. The meaningful PFS benefit of adding atezolizumab to conventional platinum-based chemotherapy favored its potential introduction as a treatment front-line option (20).

\section{Durvalumab plus tremelimumab as first-line treatment}

DANUBE is an open-label, phase 3 trial to assess durvalumab with or without tremelimumab (monoclonal antibody against CTLA-4) versus IV chemotherapy as a front-line therapy in previously untreated locally advanced or metastatic urothelial MIBC patients. A total of 1,032 patients were randomized 1:1:1 to receive either durvalumab $(\mathrm{n}=346)$ or durvalumab plus tremelimumab every 28 days for up to four cycles followed by durvalumab as a maintenance therapy every 28 days $(n=342)$ or platinumbased chemotherapy (according to cisplatin eligibility) for up to six cycles $(\mathrm{n}=344)$. In patients with PD-L1 overexpressed, median OS was 14.4 months with durvalumab as singleagent $(\mathrm{n}=209)$ versus 12.1 months with chemotherapy $(\mathrm{n}=207 ; \mathrm{P}=0.30)$. In all randomized patients, median OS was 15.1 months with durvalumab plus tremelimumab versus 12.1 months with chemotherapy $(\mathrm{P}=0.075)$. Treatmentrelated serious adverse events were reported in $9 \%$ of patients in the durvalumab group, $23 \%$ of patients in the durvalumab plus tremelimumab group, and $16 \%$ of patients in the chemotherapy group (21).

\section{Avelumab as first-line maintenance treatment}

JAVELIN Bladder 100 is an open-label, phase 3 trial, of best supportive care (BSC) with or without avelumab maintenance therapy in unresectable locally advanced or metastatic urothelial MIBC patients who derived disease response/stabilization from at least four cycles of platinumbased first-line chemotherapy. A total of 700 patients were randomized $1: 1$ to avelumab $10 \mathrm{mg} / \mathrm{kg}$ IV every 14 days + BSC (avelumab group) or BSC alone (control group). The primary endpoint was OS both in the overall population and in the PD-L1 biomarker positive population. Median OS in the overall population was 21.4 months in the avelumab group versus 14.3 months in the control group $(\mathrm{P}=0.001)$, while in the PD-L1 positive population was not estimable in the avelumab group versus 17.1 months in the control group $(\mathrm{P}<0.001)$. Grade $\geq 3$ adverse events occurred in $47 \%$ of avelumab arm patient and in $25 \%$ of control arm patient. Adverse events led to discontinuation of avelumab in $11.9 \%$ of patients. There were no immune-related deaths. The study met its primary endpoint demonstrating longer overall patient survival with first-line avelumab maintenance both in the overall population and PD-L1 positive patients (22). PD-L1 expression was assessed with the clone SP263 Ventana and considered positive if at least $25 \%$ of tumor cells stained for PD-L1, and/or at least $25 \%$ of immune cells stained for PD-L1 if more than $1 \%$ of the tumor area contained immune cells, or $100 \%$ of immune cells stained for PD-L1 if no more than $1 \%$ of the tumor area contained immune cells.

\section{Pembrolizumab as second-line treatment}

KEYNOTE-045 is an open-label, phase 3 trial, of pembrolizumab versus a chemotherapy-regimen according to investigator's choice as second-line treatment for platinum-refractory metastatic MIBC patients. OS and PFS were the two coprimary endpoints, survival measurements were done in the total population and among patients harboring a tumor PD-L $1 \geq 10 \%$. A total of 542 patients were randomized (1:1 ratio) to receive pembrolizumab $200 \mathrm{mg}$ IV (pembrolizumab group) or IV chemotherapy with docetaxel $75 \mathrm{mg} / \mathrm{mq}$, or paclitaxel $175 \mathrm{mg} / \mathrm{mg}$, or vinflunine $320 \mathrm{mg} / \mathrm{mq}$ (chemotherapy group), in 21-day cycles. In all patient population, median OS was 10.3 months for pembrolizumab group and 7.4 months for chemotherapy group $(\mathrm{P}=0.002)$; in patients with a CPS $\geq 10 \%$ (clone 22C3 Dako), median OS was 8.0 months in the pembrolizumab group and 5.2 months in the chemotherapy group $(\mathrm{P}=0.005)$. Overall, PFS did not differ between the two-study group. Pembrolizumab had a favorable toxicity profile compared with the chemotherapy agents and was associated with significantly longer OS of 
Table 1 Prospective immune-trials in bladder cancer patients

\begin{tabular}{|c|c|c|c|c|c|c|}
\hline $\begin{array}{l}\text { Trial [year] } \\
\text { (reference) }\end{array}$ & $\begin{array}{l}\text { Study } \\
\text { phase }\end{array}$ & Study drug/treatment arms & SS & $\begin{array}{l}\text { Disease } \\
\text { stage }\end{array}$ & Therapy line & Findings \\
\hline $\begin{array}{l}\text { KEYNOTE-057 } \\
{[2019](8)}\end{array}$ & 2 & Pembrolizumab monotherapy & 103 & NMIBC & BCG failure & cRR: $38 \%$ \\
\hline $\begin{array}{l}\text { ABACUS } \\
{[2018](14)}\end{array}$ & 2 & $\begin{array}{l}\text { Atezolizumab neoadjuvant } \\
\text { monotherapy }\end{array}$ & 68 & MIBC & NA & $\begin{array}{l}\text { pCR: } 31 \% \text { in all pts; pCR: } 37 \% \text { in PD-L1 } \\
\text { positive pts }\end{array}$ \\
\hline $\begin{array}{l}\text { IMvigor210 } \\
\text { [2016] (19) }\end{array}$ & 2 & $\begin{array}{l}\text { Atezolizumab monotherapy in c-DDP } \\
\text { unfit }\end{array}$ & 123 & $\mathrm{mMIBC}$ & I & $\begin{array}{l}\text { ORR: } 23 \% \text { (9\% cRR); mPFS: } 2.7 \text { mo; } \\
\text { mOS: } 15.9 \text { mo }\end{array}$ \\
\hline $\begin{array}{l}\text { IMvigor130 } \\
\text { [2020] (20) }\end{array}$ & 3 & $\begin{array}{l}\text { Atezolizumab + platinum-based } \\
\text { chemo (A) vs. Atezolizumab monotherapy } \\
\text { (B) vs. platinum-based chemo (C) }\end{array}$ & 1,213 & $\mathrm{mMIBC}$ & I & $\begin{array}{l}\text { mPFS: } 8.2 \text { vs. } 6.3 \text { mo (A vs. C) }(P=0.007) \\
\text { mOS: } 16 \text { vs. } 13.4 \text { mo (A vs. C) }(P=0.027) ; \\
\text { mOS: } 15.7 \text { vs. } 13.1 \text { mo (B vs. C) }\end{array}$ \\
\hline $\begin{array}{l}\text { JAVELIN Bladder } \\
100 \text { [2020] (22) }\end{array}$ & 3 & $\begin{array}{l}\text { Avelumab (A) maintenance } \\
+ \text { BSC vs. BSC }\end{array}$ & 700 & $\mathrm{mMIBC}$ & I & $\begin{array}{l}\text { mOS: } 21.4 \text { vs. } 14.3 \text { mo (A vs. BSC, all pts) } \\
(\mathrm{P}=0.001) ; \mathrm{mOS}: \mathrm{NE} \text { vs. } 17.1 \mathrm{mo}(\mathrm{A} \text { vs. } \\
\left.\text { BSC, } \mathrm{PD}-\mathrm{L}^{+}\right)(\mathrm{P}<0.001)\end{array}$ \\
\hline $\begin{array}{l}\text { KEYNOTE-045 } \\
{[2017](23)}\end{array}$ & 3 & $\begin{array}{l}\text { Pembrolizumab (P) vs. } \\
\text { chemo-regimen (TXT or PTX or vinflunine) }\end{array}$ & 542 & $\mathrm{mMIBC}$ & II & $\begin{array}{l}\text { mOS: } 10.3 \text { vs. } 7.4 \text { ( } P \text { vs. chemo, all pts) } \\
(P=0.002) ; \text { mOS: } 8.0 \text { vs. } 5.2(P \text { vs. chemo, } \\
\text { CPS } \geq 10 \%)(P=0.005)\end{array}$ \\
\hline $\begin{array}{l}\text { CheckMate275 } \\
{[2017](24)}\end{array}$ & 2 & Nivolumab after platinum-based chemo & 270 & $\mathrm{mMIBC}$ & II & $\begin{array}{l}\text { ORR: } 19.6 \% \text { (52/265). Responses } \\
\text { irrespective of PD-L1 }\end{array}$ \\
\hline
\end{tabular}

BCG, bacillus Calmette-Guerin; BSC, best supportive care; c-DDP unfit, cisplatin-ineligible; chemo., chemotherapy; CPS, combined positive score; CRR, complete response rate; I, first-line; II, second-line; MIBC, muscle-invasive bladder cancer; mMIBC, metastatic muscle-invasive bladder cancer; mo, months; mOS, median overall survival; mPFS, median progression-free survival; NA, not applicable; $\mathrm{NE}$, not estimable; NMIBC, non-muscle invasive bladder cancer; ORR, objective response rate; pCR, pathologic complete remission; PDL1, programmed cell-death ligand 1; pts, patients; PTX, paclitaxel; SS, sample size; TXT, docetaxel.

approximately 3 months (23).

\section{Nivolumab as second-line treatment}

The CheckMate275 was a single arm, phase 2, multicenter trial testing nivolumab in patients with metastatic or unresectable urothelial cancer who have progressed or recurred following platinum-based chemotherapy (24) (Table 1). The primary endpoint was overall objective response in all treated patients and by tumor cell PDL1 expression ( $\geq 5 \%$ and $\geq 1 \%$ ) with $28-8$ clone antibody. Confirmed objective response was achieved in 52 (19.6\%, 95\% CI: 15.0-24.9) of 265 patients. Responses were seen irrespective of tumour PD-L1 expression. In this trial, gene expression profile has been performed with unexpected results. Tumors classified as basal according to the TCGA classification had highest response to nivolumab. Moreover, the responders had high interferon- $\gamma$ gene expression, the highest CXCL9 or CXCL10 expression and the highest CD8 expression (25).

\section{Discussion}

Medical treatment for urothelial BC patients has rapidly been evolving from a platinum-based chemotherapy to immunotherapy-oriented approach at any disease stage.

Data from the neoadjuvant PURE-01 trial have demonstrated that increased PD-1/PD-L1 expression correlate with higher response to ICI therapy. This finding maintains open the debate on which is the optimal biomarker to improve decision making by physicians across daily clinical practice. Contrarily to the metastatic setting, the evaluation of PD-L1 on transurethral resection of the bladder tumour 
(TURBT) samples in the PURE-O1 trial evidenced a higher percentage of positive tumors $(>70 \%)$ and a better correlation with response (pT0 was achieved in $54.3 \%$ with PD-L1 CPS $\geq 10 \%$ compared with only $13.3 \%$ with CPS $<10 \%$ ). A possible explanation of this finding might be that TURBT specimens are more representative of the tumor tissue at the time of immunotherapy compared to primary tumors (either in RC specimens or TURBT) removed from patients many years before they become metastatic. Indeed, not only the different assays and cut-off should be taken into account when comparing different studies, but also the tissue samples used in each trial. The majority of tissue samples tested in immunotherapy trial in the metastatic setting are primary tumor and not metastatic sites.

In addition, neoadjuvant ICI therapy demonstrated to do not compromise the safety of the subsequent RC. In such context, when considering treatments combination, a phase II randomized clinical trial is evaluating neoadjuvant nivolumab plus urelumab (agonist antibody to CD137) versus nivolumab alone in MIBC patients considered as cisplatin-unfit or in those who refuse chemotherapy. The trial is currently recruiting patients (NCT02845323). Along this line, another phase 2 neoadjuvant study, currently recruiting, is testing the safety of nivolumab with or without ipilimumab (monoclonal antibody against CTLA-4) before surgery in MIBC patients not eligible for cisplatin-based chemotherapy (NCT03520491). In the adjuvant setting there is interest in clinical research as well. A phase 3 study of neo-adjuvant nivolumab and NKTR214 (a PEGylated interleukin-2 CD122-biased agonist) or nivolumab alone or standard of care in cisplatin-unfit MIBC patients is enrolling patients. A total of 540 patients are estimated to be enrolled into the study, and randomly assigned to receive neoadjuvant nivolumab plus NKTR214 followed by RC and subsequent adjuvant nivolumab plus NKTR-214 (experimental combination arm), neoadjuvant nivolumab followed by RC and continuation of adjuvant nivolumab (experimental monotherapy arm), or $\mathrm{RC}$ alone without neo-adjuvant therapy (standard of care) (NCT04209114).

Moving towards the therapeutic scenario of metastatic $\mathrm{BC}$ patients, we would like to underline that the advantage of 15.9 months as median OS observed with first-line atezolizumab in IMvigor210 phase II study in cisplatinineligible patients is doubtless encouraging, when compared with 9 months of front-line carboplatin-gemcitabine chemotherapy or with 15 months of cisplatin-based chemotherapy. As for the median PFS of 2.7 months observed in this trial, this apparently short-time outcome should not to be weighed in a negative way, because in many circumstances the full immunotherapy benefit occurs late and may be independent of the tumor response. Accordingly, the PFS estimation should not be considered as a reliable surrogate endpoint of clinical benefit under immune-based treatments. On the other hand, the 2-month immunotherapy advantage as PFS (cohort A versus cohort C) in the IMvigor130 trial seems a weak point for supporting the potential use of atezolizumab plus platinumbased chemotherapy as a front-line therapy option.

When considering treatment combinations as firstline therapy, it is worthy of mention that no benefit was reported in the phase 3 DANUBE trial with combined or single-agent immune-based regimens versus a platinumbased regimen (21).

PD-L1/PD-1 ICI agents are standard of care as secondline treatment after platinum failure, however only a minority of patients receive them and have benefit. PD-L1 expression in metastatic settings has been evaluated with different assays, cut-offs, cellular compartments, which makes the comparison among different trials challenging (Figure 1). FDA and European Medicines Agency (EMA) have recently restricted the use of the anti-PD1/PD-L1 drugs pembrolizumab and atezolizumab only in patients whose tumor is PD-L1 positive. However, to perform PD-L1 assays for both drugs, pathology laboratories need two different platforms (Dako and Ventana), often not available in the same institution (26-28). Recently IFN $-\gamma$ has been proposed as biomarker of response for ICI treatment. Levels of IFN- $\gamma$ release were investigated in non-small cell lung cancer and melanoma patients receiving immunotherapy with promising results $(29,30)$. The IFN- $\gamma$ gene expression signature has been evaluated in clinical trials on atezolizumab, nivolumab, durvalumab and pembrolizumab and demonstrated to be associated with higher ORR, PFS and OS (19,24,31,32).

Notably, the findings of avelumab in the JAVELIN Bladder 100 study with over $70 \%$ of patients alive at 12 months are therefore extremely relevant, offering for the first time a standard of care as first-line maintenance with immunotherapy after a disease control with a platinumcontaining chemotherapy. Likewise, the findings by the phase 3 KEYNOTE-045 trial of pembrolizumab versus a chemotherapy-based regimen (taxanes or vinflunine) as second-line therapy are of paramount importance. The significant 3 -month OS advantage obtained from this study overcomes the 2-month OS advantage of vinflunine 


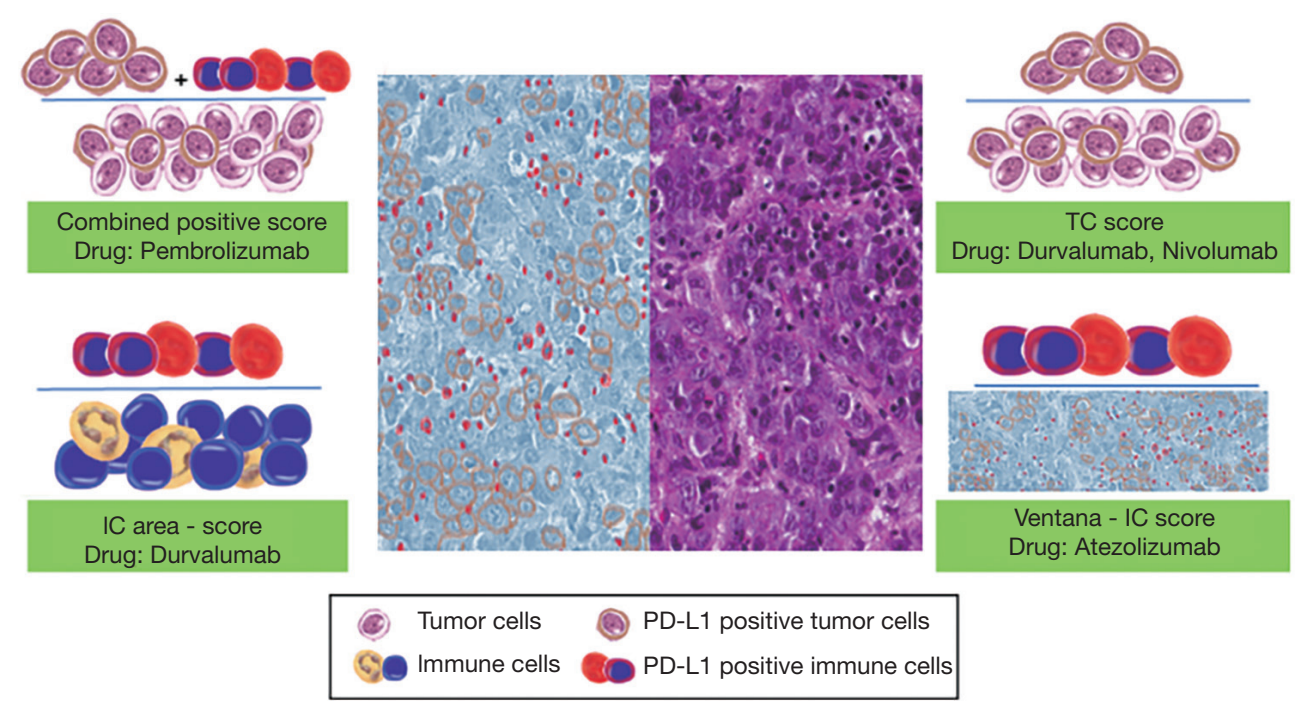

Figure 1 Illustrations of PD-L1 scoring algorithms applied in UC. PD-L1, programmed cell-death ligand 1; UC, urothelial cancer.

(approved by EMA) over BSC (10); this result is further supported by the fact that pembrolizumab has a better safety profile than that of chemotherapy agents.

\section{Conclusions}

After the FDA approval of Pembrolizumab in January 2020 large amount of real-world data of BCG-unresponsive patients treated with ICI are awaited. Considering all the data presented here and promising ongoing immune-trials, in the near future the evolving treatment paradigms of $\mathrm{BC}$ patients will increasingly incorporate immunotherapyoriented approaches in routine practice, encompassing early $\mathrm{BC}$ stages as well as advanced disease stages.

\section{Acknowledgments}

Funding: None.

\section{Footnote}

Provenance and Peer Review: This article was commissioned by the editorial office, Translational Andrology and Urology for the series "Update on Molecular Classification and Individualized Treatments of Genitourinary Tumors". The article has undergone external peer review.

Reporting Checklist: The authors have completed the
Narrative Review reporting checklist. Available at http:// dx.doi.org/10.21037/tau-20-1436

Conflicts of Interest: All authors have completed the ICMJE uniform disclosure form (available at http://dx.doi. org/10.21037/tau-20-1436). The series "Update on Molecular Classification and Individualized Treatments of Genitourinary Tumors" was commissioned by the editorial office without any funding or sponsorship. AC, ALB, MS, LC and RM served as the unpaid Guest Editors of the series. LC serves as an unpaid editorial board member of Translational Andrology and Urology from Dec 2018 to Nov 2022. The authors have no other conflicts of interest to declare.

Ethical Statement: The authors are accountable for all aspects of the work in ensuring that questions related to the accuracy or integrity of any part of the work are appropriately investigated and resolved.

Open Access Statement: This is an Open Access article distributed in accordance with the Creative Commons Attribution-NonCommercial-NoDerivs 4.0 International License (CC BY-NC-ND 4.0), which permits the noncommercial replication and distribution of the article with the strict proviso that no changes or edits are made and the original work is properly cited (including links to both the formal publication through the relevant DOI and the license). 
See: https://creativecommons.org/licenses/by-nc-nd/4.0/.

\section{References}

1. Burger M, Catto JW, Dalbagni G, et al. Epidemiology and risk factors of urothelial bladder cancer. Eur Urol 2013;63:234-41.

2. Siegel RL, Miller KD, Jemal A. Cancer statistics, 2019. CA Cancer J Clin 2019;69:7-34.

3. von der Maase H, Sengelov L, Roberts JT, et al. Longterm survival results of a randomized trial comparing gemcitabine plus cisplatin, with methotrexate, vinblastine, doxorubicin, plus cisplatin in patients with bladder cancer. J Clin Oncol 2005;23:4602-8.

4. Babjuk M, Burger M, Compérat EM, et al. European Association of Urology Guidelines on Non-muscleinvasive Bladder Cancer (TaT1 and Carcinoma In Situ) 2019 Update. Eur Urol 2019;76:639-57.

5. Raj GV, Herr H, Serio AM, et al. Treatment Paradigm Shift May Improve Survival of Patients with High Risk Superficial Bladder Cancer. J Urol 2007;177:1283-6.

6. Inman BA, Sebo TJ, Frigola X, et al. PD-L1 (B7-H1) expression by urothelial carcinoma of the bladder and BCG-induced granulomata: Associations with localized stage progression. Cancer 2007;109:1499-505.

7. Pierconti F, Raspollini MR, Martini M, et al. PD-L1 expression in bladder primary in situ urothelial carcinoma: evaluation in BCG-unresponsive patients and BCG responders. Virchows Arch 2020;477:269-77.

8. Balar AV, Kulkarni GS, Uchio EM, et al. Keynote 057: Phase II trial of Pembrolizumab (pembro) for patients (pts) with high-risk (HR) nonmuscle invasive bladder cancer (NMIBC) unresponsive to bacillus calmette-guérin (BCG). J Clin Oncol 2019;37:abstr 350.

9. Meeks JJ, Carneiro BA, Pai SG, et al. Genomic characterization of high-risk non-muscle invasive bladder cancer. Oncotarget 2016;7:75176-84.

10. Sjödahl G, Lauss M, Lövgren K, et al. A molecular taxonomy for urothelial carcinoma. Clin Cancer Res 2012;18:3377-86.

11. Lerner SP, Robertson AG. Molecular Subtypes of Nonmuscle Invasive Bladder Cancer. Cancer Cell 2016;30:1-3.

12. Meeks JJ, Lerner SP. Molecular Landscape of Non-Muscle Invasive Bladder Cancer. Cancer Cell 2017;32:550-1.

13. Robertson AG, Groeneveld CS, Jordan B, et al. Identification of Differential Tumor Subtypes of T1 Bladder Cancer. Eur Urol 2020;78:533-7.

14. Powles T, Rodriguez-Vida A, Duran I, et al. A phase II study investigating the safety and efficacy of neoadjuvant atezolizumab in muscle invasive bladder cancer (ABACUS). J Clin Oncol 2018;36:abstr 4506.

15. Necchi A, Anichini A, Raggi D, et al. Pembrolizumab as neoadjuvant therapy before radical cystectomy in patients with muscle-invasive urothelial bladder carcinoma (PURE01): An open-label, single-arm, phase II study. J Clin Oncol 2018;36:3353-60.

16. Powles T, Kockx M, Rodriguez-Vida A, et al. Clinical efficacy and biomarker analysis of neoadjuvant atezolizumab in operable urothelial carcinoma in the ABACUS trial. Nat Med 2019;25:1706-14. Erratum in: Nat Med. 2020 Jun;26(6):983 doi: 10.1038/s41591-0200923-3.

17. Necchi A, Raggi D, Gallina A, et al. Updated Results of PURE-01 with Preliminary Activity of Neoadjuvant Pembrolizumab in Patients with Muscle-invasive Bladder Carcinoma with Variant Histologies. Eur Urol 2020;77:439-46.

18. Balar AV, Galsky MD, Rosenberg JE, et al. Atezolizumab as first-line treatment in cisplatin-ineligible patients with locally advanced and metastatic urothelial carcinoma: a single-arm, multicentre, phase 2 trial. Lancet 2017;389:67-76.

19. Rosenberg JE, Hoffman-Censits J, Powles T, et al. Atezolizumab in patients with locally advanced and metastatic urothelial carcinoma who have progressed following treatment with platinum-based chemotherapy: A single-arm, multicentre, phase 2 trial. Lancet 2016;387:1909-20.

20. Galsky MD, Arija JÁA, Bamias A, et al. Atezolizumab with or without chemotherapy in metastatic urothelial cancer (IMvigor130): a multicentre, randomised, placebocontrolled phase 3 trial. Lancet 2020;395:1547-57.

21. Powles T, van der Heijden MS, Castellano D, et al. Durvalumab alone and durvalumab plus tremelimumab versus chemotherapy in previously untreated patients with unresectable, locally advanced or metastatic urothelial carcinoma (DANUBE): a randomised, open-label, multicentre, phase 3 trial. Lancet Oncol 2020;21:157488. Erratum in: Lancet Oncol. 2021 Jan;22(1):e5. doi: 10.1016/S1470-2045(20)30734-8.

22. Powles T, Park SH, Voog E, et al. Avelumab Maintenance Therapy for Advanced or Metastatic Urothelial Carcinoma. N Engl J Med 2020;383:1218-30.

23. Bellmunt J, de Wit R, Vaughn DJ, et al. Pembrolizumab as Second-Line Therapy for Advanced Urothelial Carcinoma. N Engl J Med 2017;376:1015-26. 
24. Sharma P, Retz M, Siefker-Radtke A, et al. Nivolumab in metastatic urothelial carcinoma after platinum therapy (CheckMate 275): a multicentre, single-arm, phase 2 trial. Lancet Oncol 2017;18:312-22.

25. Galsky MD, Saci A, Szabo PM, et al. Impact of zumor mutation burden on nivolumab efficacy in second-line urothelial carcinoma patients: Exploratory analysis of the phase ii checkmate 275 study. Ann Oncol 2017;28:v296-7.

26. Gevaert T, Cimadamore A, Montironi R, et al. PDL1 testing for urothelial carcinoma: Interchangeability, reliability and future perspectives. Curr Drug Targets 2021;22:162-70

27. Eckstein M, Cimadamore A, Hartmann A, et al. PD-L1 assessment in urothelial carcinoma: a practical approach. Ann Transl Med 2019;7:690.

28. Cimadamore A, Scarpelli M, Massari F, et al. Immunotherapy for urothelial cancer: from the diagnostic pathologist's point of view. Expert Opin Biol Ther 2020;20:539-44.

Cite this article as: Aurilio G, Cimadamore A, Lopez-Beltran A, Scarpelli M, Massari F, Verri E, Cheng L, Santoni M, Montironi R. Narrative review: update on immunotherapy and pathological features in patients with bladder cancer. Transl Androl Urol 2021;10(3):1521-1529. doi: 10.21037/tau-20-1436
29. Hirashima T, Kanai T, Suzuki H, et al. The Levels of Interferon-gamma Release as a Biomarker for Non-smallcell Lung Cancer Patients Receiving Immune Checkpoint Inhibitors. Anticancer Res 2019;39:6231-40.

30. Grasso CS, Tsoi J, Onyshchenko M, Abril-Rodriguez G, Ross-Macdonald P, Wind-Rotolo M, et al. Conserved Interferon- $\gamma$ Signaling Drives Clinical Response to Immune Checkpoint Blockade Therapy in Melanoma. Cancer Cell 2020;38:500-515.e3.

31. O'Donnell PH, Grivas P, Balar AV, et al. Biomarker findings and mature clinical results from KEYNOTE-052: First-line pembrolizumab (pembro) in cisplatinineligible advanced urothelial cancer (UC). J Clin Oncol 2017;35:abstr 4502.

32. Bais C, Kuziora M, Morehouse C, et al. Biologic and clinical relevance of an IFNG mRNA signature (IFNGS) and PD-L1 protein expression in tumor and immune cells in urothelial cancer (UC) patients (pts) treated with durvalumab (D). J Clin Oncol 2017;35:abstr 3037. 